\title{
Characterization of contact properties at interface between metal and graphene up to $15 \mathrm{GHz}$.
}

\author{
Shohei Kosuga ${ }^{1}$, Ryosuke Suga ${ }^{1}$, Takeshi Watanabe ${ }^{1}$, Osamu Hashimoto ${ }^{1}$, and Shinji Koh ${ }^{1}$ \\ ${ }^{1}$ Aoyama Gakuin University - Sagamihara Campus
}

June 9, 2020

\begin{abstract}
The contact properties between metal and monolayer chemical vapor deposition (CVD) graphene were investigated, and coplanar waveguides (CPWs) composed of CVD graphene-based signal lines and Au-based ground lines were fabricated. The reflection coefficients of the CPWs were experimentally measured from 1 to $15 \mathrm{GHz}$. The contact properties were represented using the equivalent circuit model, which consists of paralell contact resistance $R_{c}$ and paralell contact capacitance $C_{c}$. The calculated reflection coefficients of the model nearly agreed with the measured ones, which indicated that this model is suitable for analyzing the contact properties between metal and graphene up to $15 \mathrm{GHz}$. Bacause the impedance of $C_{c}\left(\left|1 /\left(\omega \mathrm{C}_{\mathrm{c}}\right)\right|=4.8 \times 10^{-3} \Omega\right)$ is four orders of magnitude lower than that of $R_{c}(50 \Omega)$ at $15 \mathrm{GHz}$, the current flow is more capacitive and efficient than that in the DC band. The ratio of power consumption and power storage in the microwave band to the total power consumption in the DC band decreased with increasing frequency and incresing $C_{c}$. Therefore, higher $C_{c}$ is preferable in designing microwave devices with a metal/graphene-based feeding structure, such as antennas and transmission lines.
\end{abstract}

Title pageTitle : "Characterization of contact properties at interface between metal and graphene up to 15 GHz." Author's names and affiliations :Shohei Kosuga ${ }^{1,2}$, Ryosuke Suga ${ }^{1}$, Takeshi Watanabe ${ }^{1}$, Osamu Hashimoto $^{1}$, and Shinji Koh ${ }^{1}$

${ }^{1}$ Department of Electrical Engineering and Electronics, College of Science and Engineering, Aoyama Gakuin University, 5-10-1 Fuchinobe, Chuo-ku, Sagamihara, Kanagawa 252-5258, Japan. ${ }^{2}$ Research Fellow of Japan Society for the Promotion of ScienceContact information :Shohei Kosuga kosuga@ee.aoyama.ac.jp

- AbstractThe contact properties between metal and monolayer chemical vapor deposition (CVD) graphene were investigated, and coplanar waveguides (CPWs) composed of CVD graphene-based signal lines and $\mathrm{Au}$-based ground lines were fabricated. The reflection coefficients of the CPWs were experimentally measured from 1 to $15 \mathrm{GHz}$. The contact properties were represented using the equivalent circuit model, which consists of paralell contact resistance $R_{c}$ and paralell contact capacitance $C_{c}$. The calculated reflection coefficients of the model nearly agreed with the measured ones, which indicated that this model is suitable for analyzing the contact properties between metal and graphene up to $15 \mathrm{GHz}$. Bacause the impedance of $C_{c}\left(\left|\frac{1}{\omega C_{c}}\right|=4.8 \times 10^{-3} \Omega\right)$ is four orders of magnitude lower than that of $R_{c}(50 \Omega)$ at $15 \mathrm{GHz}$, the current flow is more capacitive and efficient than that in the DC band. The ratio of power consumption and power storage in the microwave band to the total power consumption in the DC band decreased with increasing frequency and incresing $C_{c}$. Therefore, higher $C_{c}$ is preferable in designing microwave devices with a metal/graphene-based feeding structure, such as antennas and transmission lines.Keywords :graphene, contact properties, coplanar waveguide, microwave, optically transparent antennas

- Text

\section{Introduction}


Optically transparent antennas have attracted much attention because they can be located in various places without altering the landscape. Such antennas are fabricated using transparent conductive materials, such as indium tin oxide (ITO) [1], gallium-doped zinc oxide (GZO) [2], indium zinc tin oxide (IZTO) [3], and metal mesh structures [4]. We focused on graphene as an electrode material for optically transparent antennas. The high carrier mobility [5], optical transparency [6],and flexibility [7] make graphene attractive as an electrode material for optically transparent antennas. We previously reported that an invisible dipole antenna fabricated using monolayer chemical vapor deposition (CVD) graphene radiates microwaves in the microwave band [8] [9]. The optical transparency of the fabricated graphene antenna was about $97.7 \%$. Optically transparent antennas fabricated using 6-layer-stacked CVD graphene with a sheet resistance of $18 \Omega / s q$ and optical transparency of $85 \%$ has recently been reported [10]. Optically transparent microwave absorbers based on graphene [11] [12] and printed graphene-based antennas [13] [14], which are opaque to visible light, have also been reported.

The electrical contact properties at the interface between the metal-based feeding lines and graphene-based optically transparent antennas are important to obtain high radiation efficiency. The metal/graphene contact structure is the most commonly used to feed the microwave power to graphene-based antennas. In the DC band, the contact resistance at the interface between metal and graphene may significantly limit the performance of graphene-based deveices because the current injection from metal to graphene is suppressed due to the small density of states in graphene [15]. The graphene-based invisible antenna investigated in our previous studies [8] [9] consists of dipole elements fabricated using graphene as a half-wavelength dipole antenna and a coplanar waveguide (CPW) fabricated using $\mathrm{Au}$ and graphene as a feeding line. CPWs are favoarable structures for feeding graphene-based antennas because all electrodes of CPWs can be integrated on the surface side of the substrates, which are compatible with the transfer techniques of graphene and photolithography techniques. The transmission properties of CPWs fabricated with metal and graphene have been reported [16]. In the microwave band, the contact properties between metal and graphene are represented by the parallel contact resistance $R_{c}$ and parallel contact capacitance $C_{c}$ [17]. However, the properties of the metal/graphene contact in the microwave band have not been discussed and not been optimized in detail, though they are quite important to optimize the performance of the graphene-based antennas.

We focused on the properties of the metal/graphene contact in the microwave band and fabricated $\mathrm{CPWs}$ composed of $\mathrm{Au}$ electrodes and monolayer CVD graphene. To characterize the properties of the metal/graphene contact, the equivalent circuit model of the fabricated CPWs was used for the analyzing the contact properties from 1 to $15 \mathrm{GHz}$, in which the metal/graphene contact and the graphene area are represented by lumped components, which were experimentary determined. The calculated reflection coefficients for the equivalent circuit model were compared with the measured ones. Finally, we compared the impedance of $C_{c}$ and $R_{c}$.

\section{Experiments}

2. Fabrication and measurement set up 


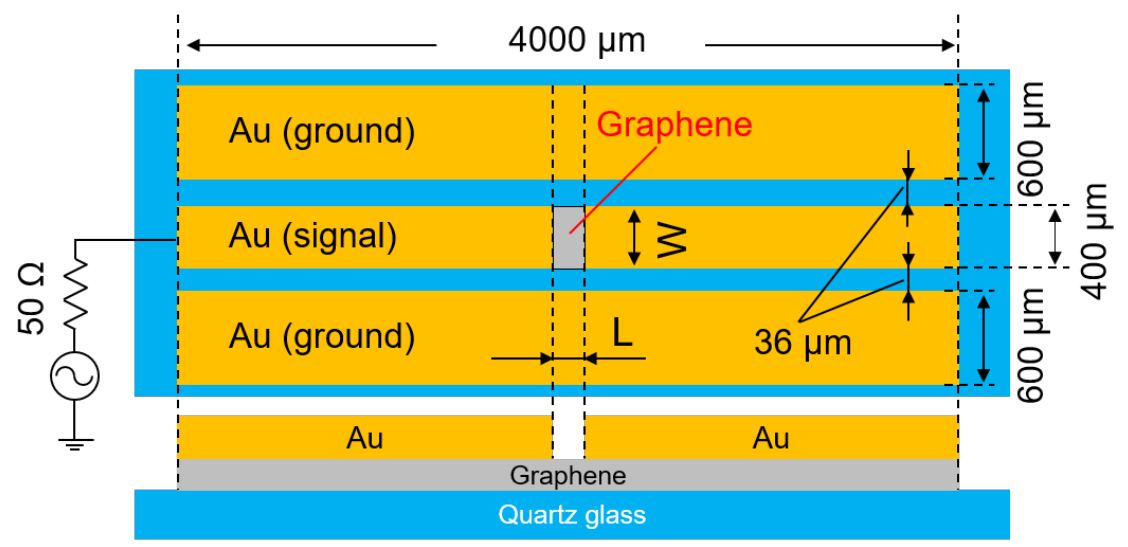

(a)

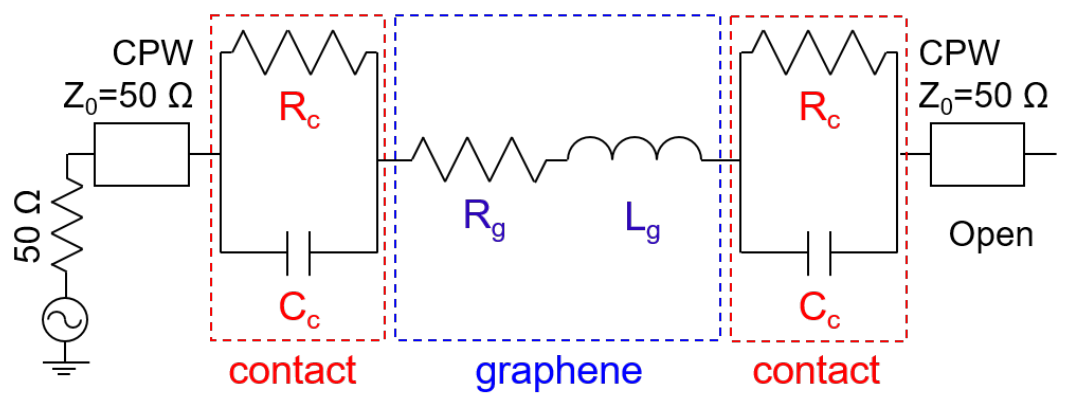

(b)

Figure 1 (a) shows the structure of one of the CPWs fabricated using monolayer CVD graphene and Au on a quartz glass substrate. Note that the graphene layers lie underneath all areas of the Au layers. In the area of monolayer CVD graphene, the width of the graphene lines $W$ was fixed $400 \mu \mathrm{m}$ and the lengthes of the graphene lines $L$ were set to 10,30 , and $50 \mu \mathrm{m}$. The characteristic impedance of an Au CPW is $50 \Omega$ and the length of the CPW is $4000 \mu \mathrm{m}$. The signal line width is $400 \mu \mathrm{m}$ and the gap between the signal line and ground is $36 \mu \mathrm{m}$. Graphene layers were grown on $\mathrm{Cu}$ foils by low-pressure CVD and transferred onto the quartz glass substrate. A CPW composed of graphene and Au was fabricated by electron beam evaporation and standard photolithography. The detailed fabrication procedure of the CPWs were reported in a previous paper [8].

The reflection coefficients of the fabricated CPWs were measured from 1 to $15 \mathrm{GHz}$ using a ground-signalground microwave probe on a wafer-probe station and a vector network analyzer. One port of the vector network analyzer was connected to one of the fabricated CPW. The terminal of the fabricated CPW was an electrical open. The measured reflection coefficients will be shown and discussed later.

\section{Equivalent circuit of the fabricated CPWs}

The contact characteristics of the metal/graphene contact were analyzed using the equivalent circuit model shown in Figure 1 (b). There are three components to this model: an Au-based CPW with characteristic impedance, contact impedance, and impedance of the graphene line. The contact impedance is represented by $R_{c}$ and $C_{c}$. We assumed that the characteristic impedance of the CPW fabricated using $\mathrm{Au} /$ graphene is 50 $\Omega$ because the contribution of the graphene underneath the Au layers is negligible and that $C_{c}$ is represented by the quantum capacitance $C_{q}$ of graphene [18]. The graphene line impedance is represented by the series resistance $R_{g}$ and series inductance $L_{g}$ of the graphene line [17]: $L_{g}$ is the kinetic inductance of the graphene 
line. It was assumed that the graphene impedance and contact impedance can be represented by lumped circuit components because $L$ is much smaller than the electrical length from 1 to $15 \mathrm{GHz}$.

1. Characterization for contact properties

2. Evaluation of impedance of graphene line and contact impedance

To quantitavely characterize $R_{g}$, we processed the monolayer CVD graphene film into a Hall bar device of a van der Pauw geometry placed on a quartz glass substrate. Hall measurements provided electrical properties, such as the sheet resistance, carrier mobillity, and carrier density of the graphene film, which are not affected by the contact resistance [19]. The measured sheet resistance, carrier mobillity, and sheet carrier density of the monolayer CVD graphene are $750 \Omega / \mathrm{sq}, 1,320 \mathrm{~cm}^{2} / \mathrm{Vs}$ and $6.4 \times 10^{12} \mathrm{~cm}^{-2}$, respectively. The Hall coefficient is postive $\left(+98 \mathrm{~m}^{2} / \mathrm{C}\right)$, inidicating that the charge carriers in the grpahene chanel are holes. According to the measured sheet resistance of the monolayer CVD graphene, the $R_{g}$ for the graphene lines of $L=10,30$, and $50 \mu \mathrm{m}$ in the CPWs can be calculated to as 19, 56, and $93 \Omega$, respectively.

The sheet impedance of graphene, $Z_{s} \Omega / s q$, can be expressed as $Z_{\mathrm{sq}}=R_{\mathrm{sq}}+j \omega L_{\mathrm{sq}}$, where $R_{s q}$ and $L_{s q}$ are the sheet resistance and sheet inductance of graphene, respectively. It should be noted that the units of $R_{s q}$ and $L_{s q}$ are defined as $\Omega / s q$ and $H / s q(=\Omega s / s q)$. Jeon et al. reported that $L_{s q}$ can be expressed as $L_{\mathrm{sq}}=\tau \mathrm{P}_{\mathrm{sq}}$, where $\tau$ is the transport relaxation time, which was assumed to be $1 \mathrm{ps}$ in a previous report [17]. We also assume that $\tau$ is 1 ps and $R_{s q}$ is determined to be $750 \Omega / s q$ in the Hall measurement. Thus, $L_{s q}$ in the graphene line in this study was estimated to be about $0.75 \mathrm{nH} / \mathrm{sq}$. Therefore, the $L_{g}$ for the graphene lines of $L=10,30$, and $50 \mu \mathrm{m}$ in the CPWs can be calculated as $0.019,0.056$, and $0.093 \mathrm{nH}$, respectively.

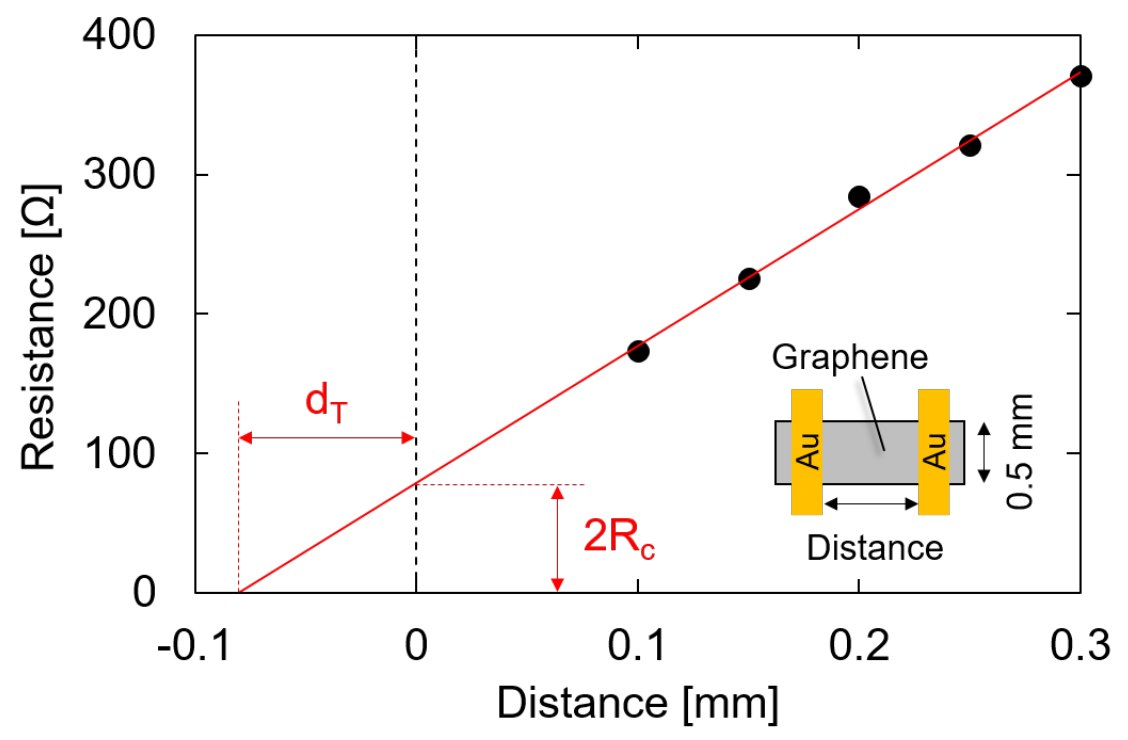

The $R_{c}$ was experimentally determined from the transfer length method (TLM) test using the TLM device structure shown in the inset of Figure 2. The TLM device consists of a monolayer CVD graphene strip and $\mathrm{Au}$ electrodes. The width of the graphene strip was fixed to $0.5 \mathrm{~mm}$, and the distances between the two adjescent $\mathrm{Au}$ electrodes were set to $0.1,0.15,0.2,0.25$, and $0.3 \mathrm{~mm}$. The contact area between the graphene strip and $\mathrm{Au}$ electrodes were set to $0.1 \times 0.5 \mathrm{~mm}^{2}$. In Figure 2, the measured resistance, including the channel resistance and contact resistances, of the graphene strip are plotted as a function of the length of the graphene strip, and the red line is the linear fitting line. For zero distance, the channel resistance vanished and the intersection point of the resistance axis corresponded to the contact resistances $2 R_{c}\left(^{\sim}\right.$ $80 \Omega$ ). Thus, the contact resistance in the TLM device was estimated to be $40 \Omega$. The contact resistivity depends on the contact width between the graphene and electrodes; hence, it can be calculated as $20 \Omega \mathrm{mm}$. 
Since the width of the graphene lines, $W$, of the CPWs was fixed to $400 \mu \mathrm{m}$, the contact resistance in the CPWs was estimated to be $50 \Omega$.

The $C_{c}$ was estimated considering the transfer area $S_{T}\left[\mathrm{~cm}^{2}\right]$ underneath the Au layer and the quantum capacitance per unit area $C_{q} \mathrm{nF} / \mathrm{cm}^{2}$ of the monolayer CVD graphene in the CPW. The $C_{c}$ can be expressed as $C_{c}=C_{q} \times S_{T} \mathrm{nF}$. At the interface between $\mathrm{Au}$ and graphene, current crowding takes place in the effective $S_{T}=d_{T} \times W$, where $d_{T}$ is the transfer length at the interface between Au and graphene. The $d_{T}$, which can be determined as the interseption point of the distance axis in Figure 2, was $\sim 00 \mathrm{~m}$ [19]. Since $W$ was fixed to $400 \mu \mathrm{m}, S_{T}$ can be calculated as $3.2 \times 10^{-4} \mathrm{~cm}^{2}$. Nagashio et al. reported that the $C_{q}$ of graphene is proportional to the Fermi energy of graphene [18] and can be expressed as

$$
C_{q}=\frac{2 e^{2} E_{F}}{\pi\left(v_{F} \hbar\right)^{2}}
$$

where $E_{F}$ is the Fermi energy, $v_{F}$ is the Fermi verocity $\left(1 \times 10^{8} \mathrm{~cm} / \mathrm{s}\right)$ and $\hbar$ is Plank's conatant. The $E_{F}$ are given by

$$
E_{F}=\hbar v_{F} \sqrt{\frac{n \pi}{\lambda}}
$$

where $n$ is the carrier density of graphene and $\lambda$ is the fitting parameter, which is generally set to 1.1 [20]. Since the measured sheet carrier density of the monolayer CVD graphene is $6.4 \times 10^{12} \mathrm{~cm}^{-2}$, the $E_{f}$ of the monolayer CVD graphene can be calculated as $\sim 0.28 \mathrm{eV}$. Therefore, the $C_{q}$ of graphene can be estimated from the above equation to be $6.7 \mu \mathrm{F} / \mathrm{cm}^{2}$. Consequently, $C_{c}$ can be calculated as $2.2 \mathrm{nF}$ using $C_{c}=C_{q} \times S_{T}$.

Discussion on contact impedance 


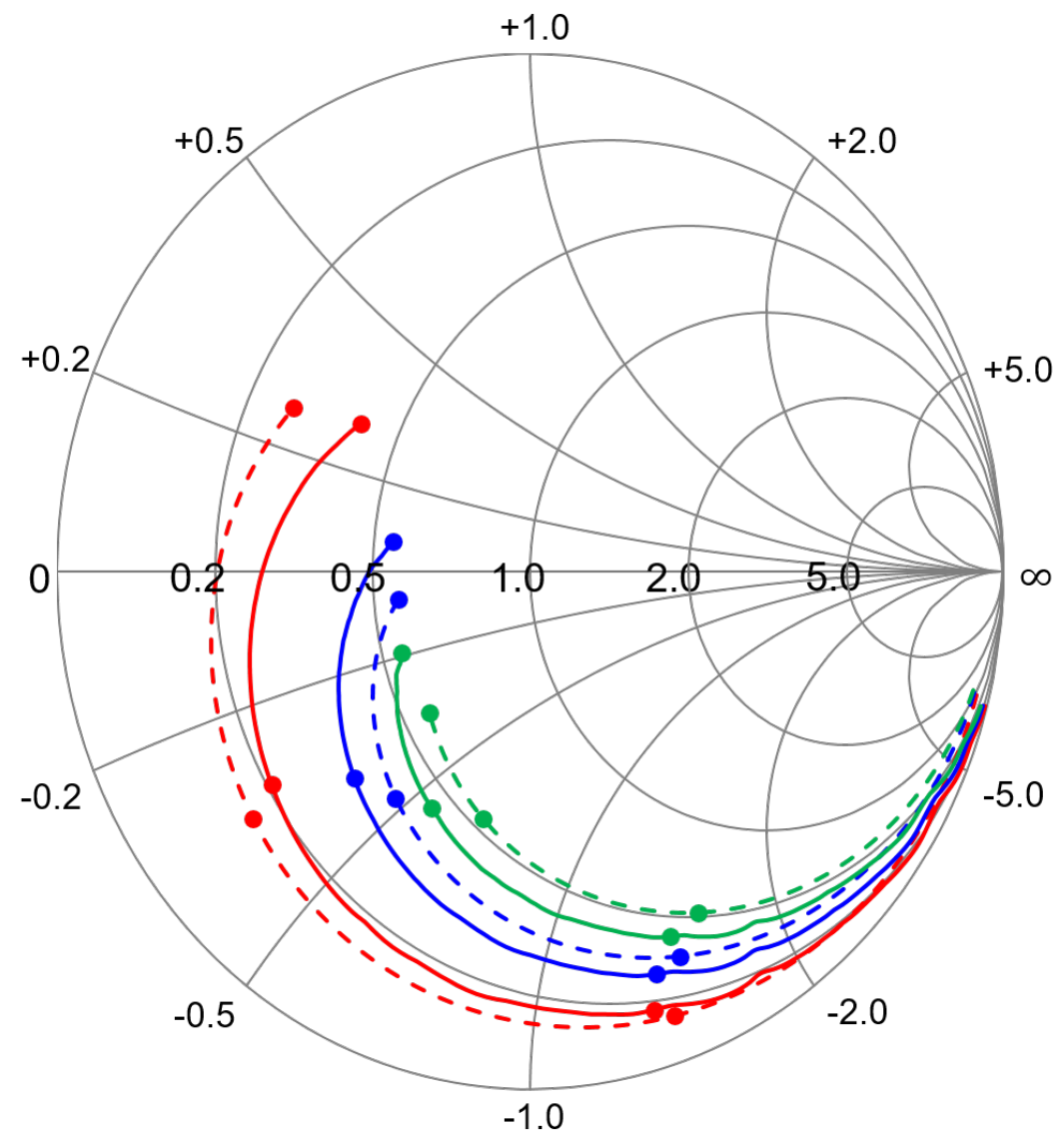

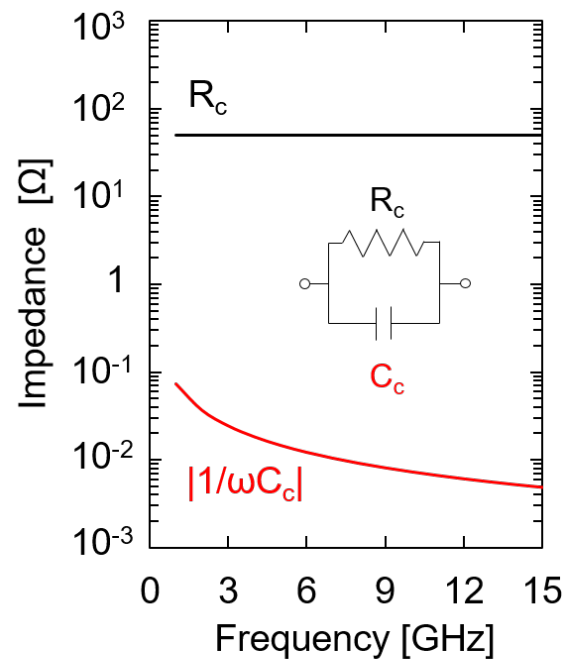

(a)

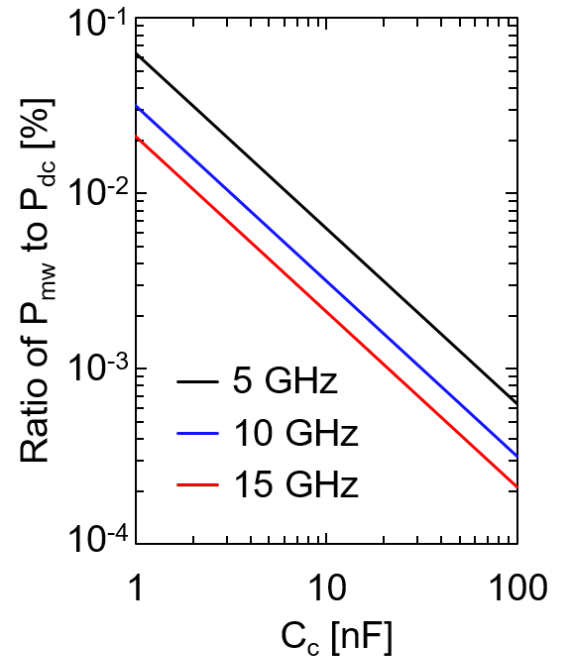

(b) 
The $R_{g}, L_{g}, R_{c}$, and $C_{c}$, which were determined in the previous section, are listed in Table I. The reflection coefficients for the equivalent circuit model were calculated using these parameters. Figure 3 shows the measured (solid lines) and calculated (dashed lines) reflection coefficients of the CPWs from 1 to $15 \mathrm{GHz}$ on a smith chart. The red, blue, and green lines correspond to the data of the reflection coefficients for CPWs with the graphene lines of $L=10,30$, and $50 \mu \mathrm{m}$. The points in Figure 3 are the measured and calculated reflection coefficients at 5, 10, and $15 \mathrm{GHz}$. As shown in Figure 3, the reflection coefficients depended on $L$. The calculated reflection coefficients for this model nearly agreed with the measured ones from 1 to $15 \mathrm{GHz}$. Therefore, this model can be applied for the analyzing the contact properties between metal and graphene up to $15 \mathrm{GHz}$. The phase angle of the reflection coefficients for the graphene lines of $L=50 \mu \mathrm{m}$ was smaller than that for graphene lines of $L=10 \mu \mathrm{m}$. We previously reported that the phase constant $\beta\left(\approx \frac{1}{2} \omega \mu_{0} \sigma\right) \mathrm{deg} / \mathrm{mmof}$ graphene is smaller than that of metal due to the lower conductivity $\sigma$ of graphene [9]. Therefore, the $\beta$ of the graphene line decreases with increasing $L$. Consequently, the contact impedance was correctly characterized by $R_{c}$ and $C_{c}$ obtained from the TLM, Hall measurement, and estimation, as shown in Table I.

We presented the contact impedance at the interface between a metal and graphene from 1 to $15 \mathrm{GHz}$. As mention the above, $R_{c}$ and $C_{c}$ were $50 \Omega$ and $2.2 \mathrm{nF}$, respectively. In Figure $4(\mathrm{a})$, the impedance of $C_{c}$ is plotted as a function of frequency. It should be that the the impedance of $C_{c}$ is represented as $\left|\frac{1}{\omega C_{c}}\right|$ and is more than two orders of magnitude lower than that of $R_{c}$ from 1 to $15 \mathrm{GHz}$. It also should be noted that the contact impedance consists of $R_{c}$ and $C_{c}$. Therefore, these results indicate that the current flows mainly not through $R_{c}$, but through $C_{c}$ in the microwave band. In the DC band, houwever, most of the current flows through $R_{c}$ because the impedance value of $C_{c}$ is approximately infinite. Therefore, it is suggested that the current flow from a metal into graphene in the microwave band is more capacitive and efficient than that in the DC band. In Figure 4 (b), the ratio of power consumption and power storage in the microwave band $P_{m w}$, to the total power consumption in the DC band $P_{d c}$, which represents the degree of energy dissipation at the metal/graphene contact, is plotted as a function of $C_{c}$. The $P_{m w} / P_{d c}$ ratio is expressed as $\frac{P_{\mathrm{mw}}}{P_{\mathrm{dc}}}=\frac{\left|\frac{1}{\omega C_{c}}\right|}{R_{c}+\left|\frac{1}{\omega C_{c}}\right|} \times 100[\%]$. The black, blue, and red lines correspond to the $P_{m w} / P_{d c}$ ratio for 5,10 , and 15 $\mathrm{GHz}$, respectively. The $P_{m w} / P_{d c}$ ratio decreased with increasing frequency and incresing $C_{c}$. Therefore, in the microwave band, $C_{c}$ is a critical factor, hence, a reduction in the impedance of $C_{c}$ should be achieved for more efficient carrier injection from metal to graphene. These results indicate that a higher $C_{c}$ is preferred in designing the feeding structure of graphene-based microwave devices such as antennas and transmission lines. For example, a larger contact area leads to a higher $C_{c}$ because of increasing $\mathrm{S}_{\mathrm{T}}$. Furthermore, increasing the $n$ of graphene may provide a higher $C_{c}$ because it is represented by $C_{q}$ of graphene, which is proportional ton .

\section{Conclusions}

The contact properties at the interface between metal and graphene in the microwave band from 1 to $15 \mathrm{GHz}$ were analyzed through experimental measurements and caluculation with an equivalent circuit model using lumped circuit components. The calculated reflection coefficients based on the lumped circuit components nearly agreed with the measured ones, which indicated that the equivalent circuit model can be applied to analyze the contact properties between metal and graphene up to $15 \mathrm{GHz}$. The impedance of $C_{c}$ was more than two orders of magnitude lower than that of $R_{c}$. This indicates that most of the carrier injection from metal to graphene flows not through $R_{c}$ but through $C_{c}$ in the microwave band, which suggests that the current flow between metal and graphene in the microwave band is more capacitive and efficient than that in the DC band. Therefore, the interface between metal and graphene with high capacitance is preferred in designing the feeding structure of graphene-based microwave devices. This work provides a building block that is essential for designing optically transparent graphene-based antennas.

- Figure legendsFigure 1: (a) Bird's eye and cross-sectional views and (b) equivalent circuit model of fabricated CPWs. Figure 2: Measured resistance extracted with TLM. Inset is schematic structure of TLM device. Figure 3: Measured (solid) and calculated (dashed) reflection coefficients with graphene lines of $\mathrm{L}=10$ (red), 30 (blue), and $50 \mu \mathrm{m}$ (green) on smith chart. Points on graph are reflection 
coefficients at 5, 10, and $15 \mathrm{GHz}$. Figure 4: (a) Impedance of parallel contact resistance $R_{c}$. and contact capacitance $C_{c}$. (b) Ratio of power consumption and power storage in microwave band $P_{m w}$ to total power consumption in DC band $P_{d c}$ for 5 (black), 10 (blue), and $15 \mathrm{GHz}$ (red). Table I: Measured and estimated parameters of CPWs.

- AcknowledgementsThis work was supported by JSPS KAKENHI (19J14640, 19K05218, and 20H02209), Aoyama Gakuin University-Supported Program "Early Eagle Program", and Nippon Sheet Glass Foundation Material Science and Engineering.

- Ethic statement We do not violate ethics.

- Conflict of Interests Authors have no conflict of interest relevant to this article.

- Literature cited[1] Guan N., Furuya H., Himeno K., Goto K., and Ito K. Basic study on an antenna made of a transparent coductive film. IEICE Trans Commun. 2007;E90-B(9):2219-2224. [2] Green R. B., Toporkov M., Ullah M. D. B., Avrutin V., Ozgur U., Morkoc H., Topsakal E. An alternative material for transparent antennas for commercial and medical applications. Microwave Opt Technol Lett. 2017;59:773-777. [3] Lee C. M., Kim Y., Kim Y., Kim I. K., Jung C. W. A flexible and transparent antenna on a polyamide substrate for laptop computers.Microwave Opt Technol Lett. 2015;57:1038-1042. [4] Clasen G. and Langley R. Meshed patch antennas. IEEE Trans Antennas Propag. 2004;52:1412 [5] Lin Y. M., Valdes-Garcia A., Han S. J., Farmer D. B., Meric I., Sun Y., Wu Y., Dimitrakopoulos C., Grill A., Avouris P., Jenkins K. A. Wafer-scale graphene integrated circuit. Science.2011;332:1294-1297. [6] Nair R. R, Blake P., Grigorenko A. N., Novoselov K. S., Booth T. J., Stauber T., Peres N. M. R., Geim A. K. Fine structure constant defines visual transparency of graphene. Science.2008;320:1308. [7] Kim K. S., Zhao Y., Jang H., Lee S. Y., Kim J. M., Kim K. S., Ahn J-Y, Kim P., Choi J-Y, Hong B. H. Large-scale pattern growth of graphene films for stretchable electrodes. Nature.2009;457:706-710. [8] Kosuga S., Suga R., Hashimoto O., Koh S. Graphene-based optically transparent dipole antenna. Appl Phys Lett.2017;110:233102. [9] Kosuga S., Suga K., Suga R., Hatanabe T., Hashimoto O., Koh S. Radiation properties of graphene-based optically transparent dipole antenna. Microwave Opt Technol Lett. 2018;60:2992-2998. [10] Grande M., Bianco G.V., Laneve D., Capazzuto P., Petruzzeli V., Scalora M., Prudenzano F., Bruno G., D’Orazio A. Optically transparent wideband CVD graphene-based microwave antennas. Appl Phys Lett. 2018;112:251103. [11] Yi D.,Wei X. C., Xu Y. L. Tunable microwave absorber based on patterned graphene. IEEE Trans Microw Theory Tech.2017;65:2819-2826. [12] Grande M., Bianco G. V., Vincenti M.A., Ceglia D.D., Capezzuto P., Petruzzelli V., Scalora M., Bruno G., D'Orazono A. Optically transparent microwave screens based on engineered graphene layers. Opt Express. 2016;24:22788-22795 [13] Huang X., Leng T., Zhu M., Zhang X., Chen J. C., Chang K. H., Aqeeli M., Geim A. K., Novoselov K. S., Hu Z. Highly flexible and conductive printed graphene for wireless wearable communications applications. Sci Rep. 2016;5:18298. [14] Sajal S., Braaten B. D., Travis T., Asif S., Schroeder M. J. Design of a coformal monopole antenna on a paper substrate using the properties of graphene-based conductors. Microwave Opt Technol Lett. 2016;59:1279-1183. [15] Nagashio K. and Toriumi A. Density-of-states limited contact resistance in graphene field-effect transistors. Jpn. J. Appl. Phys. 2011;50:070108. [16] Skulason H. S., Nguyen H. V., Guermoune A., Sridharan V., Siaj M., Caloz C., Szkopek T. 110 $\mathrm{GHz}$ measurements of large-area graphene integrated in low-loss microwave structures. Appl Phys Lett. 2011;99:153504. [17] Jeon D-Y, Lee K. L., Kim M., Kim D. C., Chung H-Y, Woo Y-S, Seo S. RadioFrequency Electrical Characteristics of Single Layer Graphene.Jpn. J. Appl. Phys. 2009;48:091601. [18] Nagashio K, Nishimura T, Toriumi A. Estimation of residual carrier density near the Dirac point in graphene through quantum capacitance measurement. Appl Phys Lett. 2013;102:173507. [19] Schroder D. K. Semiconductor Material and Device Characterization. Danvers, USA: John Wiley \& Sons; 1998. [20] Wong H. P. Carbon Nanotube and Graphene Device Physics. New York, USA: Cambridge University Press; 2011. 